\title{
Enhancing Brain Lesions during Acute Optic Neuritis and/or Longitudinally Extensive Transverse Myelitis May Portend a Higher Relapse Rate in Neuromyelitis Optica Spectrum Disorders
}

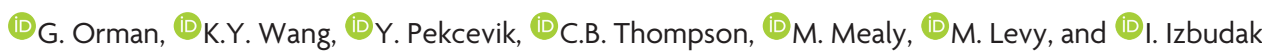

\begin{abstract}
BACKGROUND AND PURPOSE: Neuromyelitis optica spectrum disorders are inflammatory demyelinating disorders with optic neuritis and/or longitudinally extensive transverse myelitis episodes. We now know that neuromyelitis optica spectrum disorders are associated with antibodies to aquaporin-4, which are highly concentrated on astrocytic end-feet at the blood-brain barrier. Immune-mediated disruption of the blood-brain barrier may manifest as contrast enhancement on brain MR imaging. We aimed to delineate the extent and frequency of contrast enhancement on brain MR imaging within 1 month of optic neuritis and/or longitudinally extensive transverse myelitis attacks and to correlate contrast enhancement with outcome measures.
\end{abstract}

MATERIALS AND METHODS: Brain MRIs of patients with neuromyelitis optica spectrum disorders were evaluated for patterns of contrast enhancement (periependymal, cloudlike, leptomeningeal, and so forth). The Fisher exact test was used to evaluate differences between the proportion of contrast enhancement in patients who were seropositive and seronegative for aquaporin- 4 antibodies. The Mann-Whitney test was used to compare the annualized relapse rate and disease duration between patients with and without contrast enhancement and with and without seropositivity.

RESULTS: Brain MRIs of 77 patients were evaluated; 59 patients (10 males, 49 females) were scanned within 1 month of optic neuritis and/or longitudinally extensive transverse myelitis attacks and were included in the analysis. Forty-eight patients were seropositive, 9 were seronegative, and 2 were not tested for aquaporin-4 antibodies. Having brain contrast enhancement of any type during an acute attack was significantly associated with higher annualized relapse rates $(P=.03)$ and marginally associated with shorter disease duration $(P=.05)$. Having periependymal contrast enhancement was significantly associated with higher annualized relapse rates $(P=.03)$.

CONCLUSIONS: Brain MRIs of patients with neuromyelitis optica spectrum disorders with contrast enhancement during an acute relapse of optic neuritis and/or longitudinally extensive transverse myelitis are associated with increased annual relapse rates.

ABBREVIATIONS: AQP4 = aquaporin-4; $A R R=$ annualized relapse rate; $C E=$ contrast enhancement; $I g G=$ immunoglobulin $\mathrm{G}$; LETM = longitudinally extensive transverse myelitis; $\mathrm{NMO}=$ neuromyelitis optica; NMOSD = NMO spectrum disorders; $\mathrm{ON}=$ optic neuritis

$\mathbf{N}$ euromyelitis optica (NMO) is an inflammatory demyelinating disorder of the central nervous system, ${ }^{1}$ characterized by recurrent episodes of longitudinally extensive transverse myelitis (LETM) and/or optic neuritis (ON). ${ }^{2}$ Discovery of an NMO-specific autoantibody, NMO-immunoglobulin G (IgG), and its tar-

Received December 22, 2016; accepted January 14, 2017.

From the Division of Neuroradiology (G.O., Y.P., I.I.), Russell H. Morgan Department of Radiology, and Department of Neurology (M.M., M.L.), The Johns Hopkins University School of Medicine, Baltimore, Maryland; Department of Radiology (K.Y.W.), Baylor College of Medicine, Houston, Texas; and Biostatistics Center (C.B.T.), Johns Hopkins Bloomberg School of Public Health, Baltimore, Maryland.

Carol Thompson received a National Institutes of Health-Clinical and Translational Science Awards grant 1ULITR001079 (to Johns Hopkins). Maureen Mealy received honoraria from the International Organization of Multiple Sclerosis Nurses and EMD Serono. Michael Levy received research support from the Guthy-Jackson Charitable Foundation, ViroPharma, Acorda Therapeutics, Sanofi, Neuralstem, and Genentech and serves as a consultant for Chugai Pharmaceuticals, GlaxoSmithKline, Alexion Pharmaceuticals, and Medlmmune. get autoantigen, aquaporin-4 (AQP4), have differentiated NMO from multiple sclerosis as a distinct disease entity. ${ }^{3}$ Moreover, given the high specificity of AQP4-IgG serology for clinically diagnosed NMO, such seropositivity was incorporated into the revised diagnostic criteria for NMO in 2006. ${ }^{1}$ The term "NMO spectrum disorders" (NMOSD) was introduced in 2007 to encompass broader phenotypes, including seropositive patients with coexisting autoimmune disorders and patients with limited or inaugural

\footnotetext{
Paper previously presented in part as an oral presentation at: Annual Meeting of the American Society of Neuroradiology and the Foundation of the ASNR Symposium, April 25-30, 2015, Chicago, Illinois. The presentation number was O-444.

Please address correspondence to Izlem Izbudak, MD, Division of Neuroradiology, Russell H. Morgan Department of Radiology, The Johns Hopkins University School of Medicine, 600 N Wolfe St, Phipps B-126B, Baltimore, MD 21287; e-mail: iizbuda1@jhmi.edu

-* Indicates open access to non-subscribers at www.ajnr.org

http://dx.doi.org/10.3174/ajnr.A5141
} 


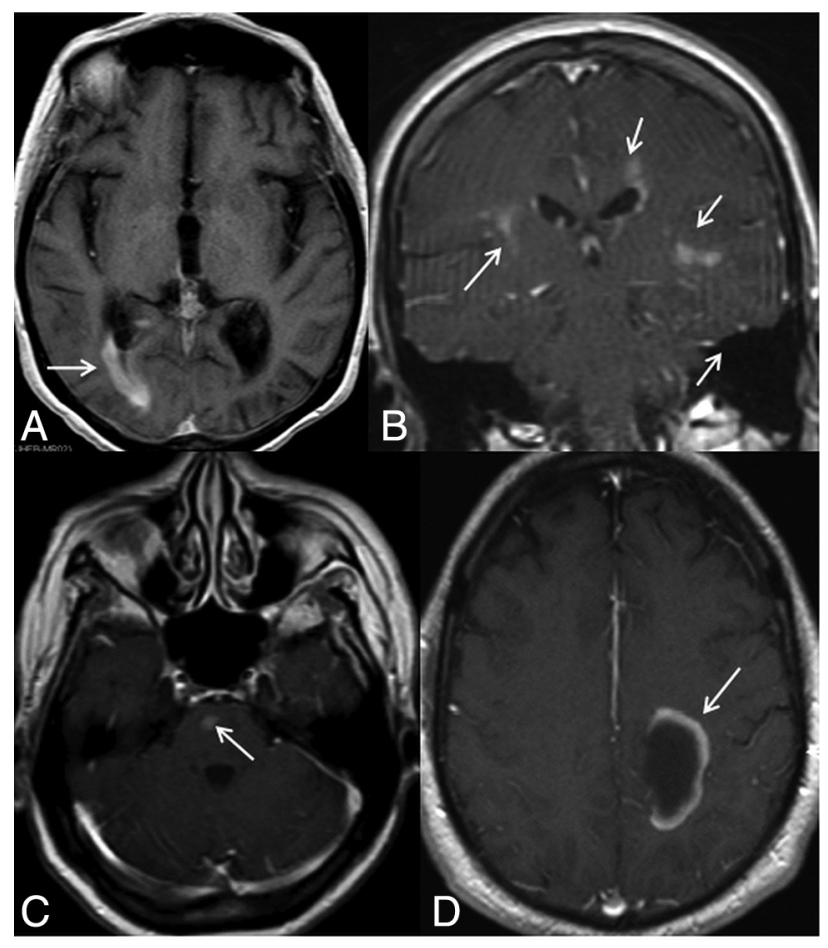

FIG 1. Different contrast-enhancement patterns are shown in this figure. A, A periependymal linear enhancement pattern can be seen surrounding the lateral, third or fourth ventricles, and/or cerebral aqueduct. Here we see right posterior periventricular enhancement (arrow). B, Periependymal enhancement and inhomogeneous, subtle parenchymal enhancement with ill-defined margins, so-called cloudlike enhancement (arrows). C, Isolated enhancement (arrow), D, Incomplete ring enhancement (arrow).

forms of NMO. ${ }^{4}$ The terms NMO and NMOSD were unified under a revised NMOSD definition in $2015 .^{5}$ The unifying NMOSD diagnostic criteria allowed the diagnosis of NMOSD in patients without clinical involvement of the optic nerves or the spinal cord and stratified the diagnosis according to those with or without AQP4-IgG positivity.

While NMO was traditionally thought to be a disease exclusively involving the optic nerves and spinal cord, imaging abnormalities within the brain have been reported in a significant proportion of patients seropositive for AQP4-IgG, in regions with both high ${ }^{6,7}$ and low AQP4 expression. ${ }^{8}$ Lesions involving the diencephalon, area postrema, corpus callosum, hemispheric white matter, and corticospinal tracts have been reported. ${ }^{8}$ Specific patterns of contrast enhancement (CE) within the brain have also been reported in NMO, including pencil-thin, ${ }^{9}$ cloudlike, ${ }^{10}$ leptomeningeal, ${ }^{11}$ and perivascular enhancement (Figs 1 and 2). ${ }^{12}$ The current literature suggests a relatively low incidence of contrastenhancing brain lesions in NMO. ${ }^{9-11,13-18}$

However, in a large proportion of brain MRIs in those studies, whether they were acquired during an acute phase of the disease versus at any time point was not specified. Furthermore, the incidence of contrast-enhancing lesions in the brain during acute relapses of ON and/or LETM in patients with NMOSD has not been examined before, to our knowledge. Prior studies investigating predictors of relapse in patients with NMOSD have addressed factors that are either clinical or biochemical in nature, including AQP4-IgG seropositivity, ${ }^{19}$ female sex, ${ }^{20-22}$ and older age of on-

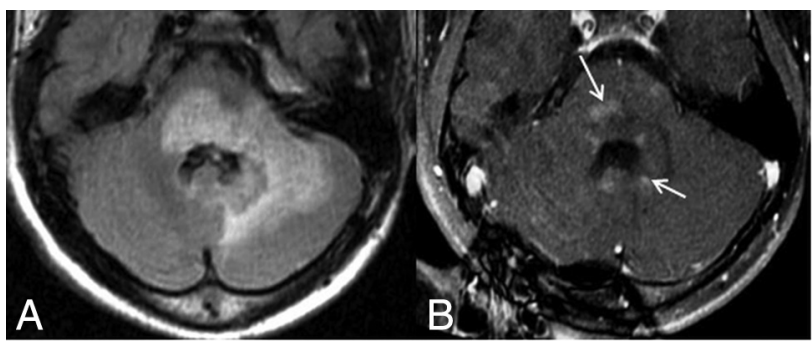

FIG 2. A 21-year-old male patient diagnosed with neuromyelitis optica. He initially presented with longitudinally extensive transverse myelitis when he was 12 years of age. MR imaging was performed at 14 years of age within 1 month of an acute LETM attack. Axial FLAIR image (A) shows a large region of increased signal abnormality within the pons, extending into the left middle cerebellar peduncle with expansion of the pons itself and the cerebellar hemisphere. Postcontrast T1-weighted image $(B)$ shows cloudlike contrast enhancement (arrows).

set. ${ }^{23}$ In contrast, no MR imaging parameters have been shown to be associated with disease outcome.

In the current study, we aimed to delineate the extent and frequency of $\mathrm{CE}$ in the brain during acute attacks of $\mathrm{ON}$ and/or LETM. We also sought to determine whether detection of brain $\mathrm{CE}$ was associated with specific outcome measures, including disease duration and the annualized relapse rate (ARR).

\section{MATERIALS AND METHODS}

\section{Patients}

A retrospective chart review was performed to identify patients with contrast-enhancing brain lesions between September 2001 and November 2013 at the Johns Hopkins NMO center. All patients identified were diagnosed with NMO or NMOSD based on the Wingerchuk et al $2006^{1}$ or $2007^{4}$ revised criteria, respectively. ${ }^{5}$ Institutional review board approval was obtained for the study. Electronic patient records were reviewed for demographic information, history of relapse, AQP4-IgG status, age at diagnosis, age at last follow-up, and the number of relapses.

\section{Neuroimaging}

MR imaging examinations were performed by using either $1.5 \mathrm{~T}$ or 3T scanners (Philips Healthcare, Best, the Netherlands; GE Healthcare, Milwaukee, Wisconsin; and Siemens, Erlangen, Germany). T1WI, fast spin-echo T2WI, fast spin-echo FLAIR, and postgadolinium T1WIs were performed. A gadolinium contrast agent of $0.1 \mathrm{~mL} / \mathrm{kg}$ was intravenously administered followed by a 20-mL saline injection. T1-weighted axial and coronal images were acquired without any delay after intravenous injection. The sagittal T1WIs were obtained with the following parameters: TR range $=520-696 \mathrm{~ms}$, TE range $=4.6-14 \mathrm{~ms}$, matrix size range $=$ $192 \times 192$ to $512 \times 196$, FOV range $=190 \times 190 \mathrm{~mm}$ to $240 \times$ $240 \mathrm{~mm}$, section thickness $/$ spacing range $=1 / 1$ to $5 / 7 \mathrm{~mm}$. Axial T2WI was performed with the following parameters: TR range $=$ $2500-7000 \mathrm{~ms}$, TE range $=83-112 \mathrm{~ms}$, matrix size range $=256 \times$ 184 to $448 \times 335$, FOV range $=159 \times 200 \mathrm{~mm}$ to $240 \times 240 \mathrm{~mm}$, section thickness/spacing range $=2 / 2$ to $5 / 5 \mathrm{~mm}$. A FLAIR sequence was obtained with the following parameters: $\mathrm{TR}=6000$ $\mathrm{ms}, \mathrm{TE}=120 \mathrm{~ms}$, TI $=2000 \mathrm{~ms}$, section thickness $=5 \mathrm{~mm}$, $\mathrm{FOV}=23 \mathrm{~cm}$, matrix size $=256 \times 256$.

All brain MRIs were evaluated in consensus by 2 radiologists, a 
Table 1: Association between the proportion of patients with CE and the presence of AQP4-IgG seropositivity ${ }^{a}$

\begin{tabular}{lccc}
\hline AQP4-IgG & CE Present & CE Absent & No. of Patients (Total) \\
\hline Positive & $31(64.6 \%)$ & $17(35.4 \%)$ & 48 \\
Negative & $4(55.6 \%)$ & $5(44.4 \%)$ & 9 \\
Total & $36(63.2 \%)^{\mathrm{b}}$ & $21(36.8 \%)^{\mathrm{b}}$ & $57(2$ not tested) \\
\hline
\end{tabular}

${ }^{a}$ Fisher exact test, $P=.7$

${ }^{\mathrm{b}}$ One patient was not tested.

Table 2: Comparison of disease duration and ARR between patients with and without CE during an acute attack

\begin{tabular}{lccc}
\hline & CE Present & CE Absent & $\begin{array}{c}\boldsymbol{P} \\
\text { Value }\end{array}$ \\
\hline $\begin{array}{l}\text { Disease duration } \\
\text { (mean) (yr) }\end{array}$ & $4.76 \pm 4.81(n=21)$ & $7.26 \pm 5.75(n=38)$ & .05 \\
ARR (mean) & $1.15 \pm 0.73(n=21)$ & $0.73 \pm 0.52(n=38)$ & $.03^{\mathrm{a}}$ \\
\hline a $P<.05$, based on Mann-Whitney test. &
\end{tabular}

board-certified neuroradiologist (I.I.) and a radiologist (G.O.), with 10 and 4 years of experience, respectively. All patients had at least 1 brain MR imaging performed at our institution. Brain MRIs acquired within 1 month of the onset of the relapse were classified as imaging during an acute LETM and/or ON attack. CE was evaluated by using postgadolinium T1-weighted images. Brain CE was categorized in 6 specific patterns of enhancement: periependymal, cloudlike, leptomeningeal, isolated, ring, or other (Figs 1 and 2).

\section{Statistical Analysis}

The Fisher exact test was used to evaluate the difference between the proportions of patients with $\mathrm{CE}$ who were seropositive versus seronegative. A nonparametric Mann-Whitney test was used to compare the ARR and disease duration between those with and without $\mathrm{CE}$. Regression analyses of the ARR with and without $\mathrm{CE}$ were also performed, with and without adjusting for age, sex, race, and AQP4-status. $P$ values $<.05$ were considered statistically significant and were not adjusted for multiple analyses.

\section{RESULTS}

Brain MRIs of 77 patients (11 males, 66 females) were evaluated for contrast enhancement. Fifty-nine patients (10 males, 49 females) underwent brain MR imaging within 1 month of the onset ON and/or LETM attack and were included in the final analysis. The mean age of patients was 47.8 years (range, $6-78$ years). There were 35 African-American, 18 white, and 6 Hispanic (individuals from Mexico) individuals. Forty-eight patients were AQP4-IgG seropositive, 9 were seronegative, and the AQP4-IgG status was not checked in 2 of them. The ARR was not available for 1 patient.

Table 1 depicts the proportions of patients with CE in those with or without AQP4-IgG seropositivity during acute attacks. The Fisher exact test did not demonstrate significantly different proportions of $\mathrm{CE}$ in patients with or without AQP4-IgG seropositivity during acute attacks $(P=.7)$. No significantly different proportions were noted when stratified by specific enhancement patterns $(P=.7$, data not shown).

Tables 2 and 3 depict the association between the detection of CE during an acute phase and either disease duration or ARR. When imaged during the acute phase, patients demonstrating periependymal CE had significantly higher ARRs compared with those without periependymal CE $(P=.03)$. More-
Table 3: Comparison of disease duration and ARR between patients with and without PCE during an acute attack

\begin{tabular}{lccc}
\hline & PCE Present & PCE Absent & P Value \\
\hline $\begin{array}{l}\text { Disease duration } \\
\text { (mean) }(y r)\end{array}$ & & \\
ARR $($ mean $)$ & $1.30 \pm 0.81(n=21)$ & $7.26 \pm 5.75(n=38)$ & .05 \\
\hline $\begin{array}{l}\text { Note:-PCE indicates periependymal contrast enhancement. } \\
\text { a } P<.05, \text { based on the Mann-Whitney test. }\end{array}$ &
\end{tabular}

Table 4: Distribution of brain CE patterns among 59 patients with ON and/or LETM

Type of CE No. of Patients

\begin{tabular}{lc}
\hline Periependymal & 14 \\
Cloudlike & 7 \\
Leptomeningeal & 2 \\
Isolated & 4 \\
Ring & 2 \\
Other & 2 \\
Absent & 28 \\
\hline
\end{tabular}

over, patients demonstrating any type of $\mathrm{CE}$ during the acute phase had significantly higher ARRs $(P=.03)$ than those without.

On the basis of the regression analyses, the unadjusted difference in ARRs between those with periependymal CE and those without it was 0.56 ( $95 \% \mathrm{CI}, 0.07-1.05 ; P=.03)$. After we adjusted for age, sex, race, and AQP4 status, the difference was 0.60 ( $95 \% \mathrm{CI}, 0.08-1.13 ; P=.03)$. The unadjusted difference in ARRs between those with any CE and without was 0.42 (95\% CI, $0.04-$ $0.80 ; P=.03)$. After we adjusted for age, sex, race, and AQP4 status, the difference was $0.41(95 \% \mathrm{CI}, 0.02-0.81 ; P=.04)$.

Table 4 shows the distribution of brain CE patterns among 59 patients who were scanned within 1 month of an ON and/or LETM attack. Brain CE was categorized and evaluated in 6 specific patterns of enhancement in the beginning of the study: periependymal, cloudlike, leptomeningeal, isolated, ring, or other (Figs 1 and 2). After excluding MRIs that were not obtained within 1 month of ON and/or LETM attack from the final analysis, we regrouped MRIs into 2 groups: a group with periependymal CE and a group with any type of CE. MRIs of 14 patients showed periependymal CE, and 21 patients showed any type of CE within 1 month of ON and/or LETM attacks.

\section{DISCUSSION}

The current literature on NMO is limited in its description of neuroimaging features that may predict the outcome of disease. ${ }^{24}$ Most asymptomatic NMO brain lesions have not been shown to demonstrate enhancement, and the frequency of acute lesion-associated enhancement remains to be determined. ${ }^{23}$ This study demonstrates that approximately $63 \%$ of patients during an acute attack of ON and/or LETM may also show CE within the brain parenchyma. CE within the brain, when identified during an acute phase, is associated with a significantly increased ARR. The relapse rate during the first 2 years of the disease strongly determines the risk of an unfavorable outcome as defined by severe disability or death. ${ }^{25}$ Brain enhancement in patients during an acute ON and/or LETM may reflect a more severe underlying disease process compared with those without brain CE.

We found no significant difference in the propensity for CE in patients who were AQP4-IgG seropositive (64.6\%) and seronegative (55.6\%) $(P=.7$, Table 1). CE patterns of brain lesions in the 
current literature were described mostly in patients seropositive for AQP4-IgG and have been reported to range from 3\% to 56\%, excluding small case reports. ${ }^{3,7,9,10,13,16,17,26}$ Our study revealed a much higher proportion of CE, approximating $64.6 \%$ and $55.6 \%$ in patients seropositive and seronegative, respectively. A study investigating contrast-enhancing LETM lesions reported CE in $94 \%$ and $71 \%$ of seropositive and seronegative patients, respectively, though the authors did not specify the location of CE as within either the brain or spinal cord. ${ }^{27}$ To our knowledge, the current study is the largest cohort to report the frequency of contrast-enhancing brain lesions in both seropositive and seronegative patients during active $\mathrm{ON}$ and/or LETM relapse of NMO. The small sample of patients seronegative for AQP4-IgG in the current study may be contributing to lack of detection for a significant difference in the proportion of CE seen in seropositive and seronegative patients; however, that might be the case in prior studies failing to show a difference as well. Nevertheless, the number of patients seronegative for AQP4-IgG is always low compared with those who are seropositive; therefore, multi-institutional studies are needed to increase the sample size.

That AQP4 is highly expressed on astrocytic foot processes at the $\mathrm{BBB}$ and contributes to the maintenance of $\mathrm{BBB}$ integrity is welldescribed. ${ }^{3,28,29}$ Binding of AQP4-IgG to AQP4 in vitro has been shown to alter $\mathrm{BBB}$ permeability and astrocyte killing. ${ }^{30}$ Disruption of the $\mathrm{BBB}$ manifests as $\mathrm{CE}$ on brain MR imaging. ${ }^{31}$ Periependymal white matter is one of the most AQP4-rich regions of the brain; hence, the high prevalence of periependymal contrast enhancement in our cohort. Furthermore, AQP4-IgG is thought to be pathogenic only in proximity to CNS parenchyma, as evidenced by NMO-like histopathology in animal models in those that received direct administration of AQP4-IgG into the CNS. In contrast, peripheral administration had no effect. ${ }^{32}$ The presence and levels of AQP4-IgG in CSF are associated and correlated with those in serum during acute relapses. ${ }^{33,34}$ For example, AQP4-IgG is detectable in the CSF of most seropositive patients with serum titers of $>1: 250$ during an acute relapse. ${ }^{35}$ Moreover, the amount of CSF AQP4-IgG is correlated with astrocyte damage and BBB breakdown. ${ }^{34}$ Therefore, it is possible that those with lower serum titers or those not in acute relapse in the current study may not have detectable or significant CSF levels of AQP4-IgG to lead to the BBB disruption and consequent CE. Most interesting, there have been reports of patients with NMOSD who are AQP4-IgG positive in the serum for many years before the onset of symptomatic disease. ${ }^{36}$ The poor correlation between the presence and level of serum and CSF titers of AQP4-IgG may be contributing to the lack of significantly different proportions of CE in seropositive and seronegative patients in our study. It may also be contributing to the high interstudy variability in the reports of the percentage of CE observed in patients with NMOSD. 3,7,9,10,13,16,17,26,27

Most important, the association between CE during the acute phase and ARR may be confounded because those who underwent brain MRIs during an acute phase may have warranted more immediate imaging because they may have been inherently sicker. Findings may be further confounded by other clinical characteristics. For example, longer intervals between the first and second attack, ${ }^{37}$ older age at onset, ${ }^{23}$ patients of African origin, ${ }^{38}$ female sex, ${ }^{20-22}$ and AQP4-IgG seropositivity ${ }^{19}$ are associated with worse outcomes and/or higher relapse rates. However, other studies failed to find AQP4-IgG status as a predictor of outcome. Jarius et $\mathrm{al}^{39}$ found that AQP4-IgG status did not differ significantly with regard to time to relapse or ARR. Jiao et $\mathrm{al}^{40}$ found that the effect of seropositive status on the relapse rate and disability outcome did not differ. Responses to plasmapheresis based on AQP4IgG were also not significantly different. ${ }^{41}$ Regardless of the discrepant prognostic findings of AQP4-IgG in the existing literature, in our study, a periependymal pattern of CE and the presence of any pattern of CE in the acute phase remained significant predictors of higher ARRs after adjusting for AQP4 status, as well as age, sex, and race, on multivariable analysis.

Enhancement patterns of brain lesions in NMO have some unique features and sometimes, in the presence of characteristic T2 lesions, might aid in making a specific diagnosis. Patchy CE with blurred margins, so-called "cloudlike enhancement," is the most commonly reported enhancement pattern in the literature. ${ }^{10}$ More recently, linear periependymal CE, so called "pencil-thin enhancement," and leptomeningeal CE were proposed as more specific patterns than cloudlike enhancement. ${ }^{9,11}$ Isolated CE and ring and open-ring CE are considered specific to MS, and they are rarely seen in patients with NMOSD. However, although rare, these intense, well-defined CE patterns have been described before, especially in seronegative patients with NMOSD. ${ }^{18}$

The main limitation of our study is the retrospective design, and factors that have been described in the literature associated with outcomes such as seropositivity status, sex, race, and age at onset may be potential confounders and were not accounted for. There is a possible selection bias based on a group of patients with NMOSD who required brain MR imaging, which may reflect a different subpopulation than that not requiring brain MRIs. The threshold of 1 month as the criterion for an acute attack may be arbitrary, given the lack of information in records available to more accurately assess the patients' clinical statuses and may thus misrepresent these statuses in the current study. Furthermore, the current study was originally conducted before the introduction of the more inclusive revised diagnostic criteria for NMOSD of 2015. ${ }^{5}$ Rather, included patients were based on the 2006 diagnostic criteria; therefore, the current study does not account for patients who may now qualify as diagnostic for NMOSD under the 2015 criteria.

\section{CONCLUSIONS}

Detection of CE in postgadolinium T1-weighted brain imaging within 1 month of onset of an acute ON and/or LETM is associated with higher ARRs. CE is an important marker reflecting the underlying pathogenic process of NMOSD. Although no significant association was found between CE and AQP4-IgG serostatus, the strong interplay among the BBB disruption, AQP4-IgG deposition, and CE warrants further investigation with a larger multicenter cohort to determine the prognostic role that CE may play as a predictor of outcome and its correlation with clinical severity.

Disclosures: Carol B. Thompson-RELATED: Grant: National Institutes of HealthClinical and Translational Science Awards IULITR001079 to Johns Hopkins, Comments: The grant provides limited free statistical consultation to researchers. ${ }^{*} \mathrm{Mau}-$ reen Mealy_UNRELATED: Payment for Lectures Including Service on Speakers 
Bureaus: Consortium of Multiple Sclerosis Centers, Comments: honoraria. Michael Levy-UNRELATED: Grant: National Institutes of Health, Genzyme/Sanofi, Alnylam Pharmaceuticals, Alexion Pharmaceuticals, Medlmmune, Shire*; Consulting Fee or Honorarium: Genzyme/Sanofi, Alexion Pharmaceuticals; Fees for Participation in Review Activities such as Data Monitoring Boards, Statistical Analysis, Endpoint Committees, and the Like: Quest Diagnostics; UNRELATED: Board Membership: Acorda Therapeutic, Alexion Pharmaceuticals, Chugai Pharmaceuticals, Asterias Biotherapeutics; Payment for Development of Educational Presentations: Quest Diagnostics. Izlem IzbudakUNRELATED: Consultancy: Alexion Pharmaceuticals, Comments: consulting neuroradiologist for the NMO 301 and NMO 302 PREVENT trial sponsored by Alexion Pharmaceuticals; Grants/Grants Pending: Siemens, Comments: DTI of the spinal cord in compressive myelopathy, Principal Investigator of the grant*; Other: Biogen, Comments: neuroradiologist of MS PATHS study partner institute. * Money paid to the institution.

\section{REFERENCES}

1. Wingerchuk DM, Lennon VA, Pittock SJ, et al. Revised diagnostic criteria for neuromyelitis optica. Neurology 2006;66:1485-89 CrossRef Medline

2. Wingerchuk DM, Hogancamp WF, O'Brien PC, et al. The clinical course of neuromyelitis optica (Devic's syndrome). Neurology 1999; 53:1107-14 CrossRef Medline

3. Lennon VA, Wingerchuk DM, Kryzer TJ, et al. A serum autoantibody marker of neuromyelitis optica: distinction from multiple sclerosis. Lancet 2004;364:2106-12 CrossRef Medline

4. Wingerchuk DM, Lennon VA, Lucchinetti CF, et al. The spectrum of neuromyelitis optica. Lancet Neurol 2007;6:805-15 CrossRef Medline

5. Wingerchuk DM, Banwell B, Bennett JL, et al; International Panel for NMO Diagnosis. International consensus diagnostic criteria for neuromyelitis optica spectrum disorders. Neurology 2015;85:177-89 CrossRef Medline

6. Pittock SJ, Weinshenker BG, Lucchinetti CF, et al. Neuromyelitis optica brain lesions localized at sites of high aquaporin 4 expression. Arch Neurol 2006;63:964-68 CrossRef Medline

7. Pittock SJ, Lennon VA, Krecke K, et al. Brain abnormalities in neuromyelitis optica. Arch Neurol 2006;63:390-96 CrossRef Medline

8. Kim HJ, Paul F, Lana-Peixoto MA, et al; Guthy-Jackson Charitable Foundation NMO International Clinical Consortium \& Biorepository. MRI characteristics of neuromyelitis optica spectrum disorder: an international update. Neurology 2015;84:1165-73 CrossRef Medline

9. Banker P, Sonni S, Kister I, et al. Pencil-thin ependymal enhancement in neuromyelitis optica spectrum disorders. Mult Scler 2012; 18:1050-53 CrossRef Medline

10. Ito S, Mori M, Makino T, et al. “Cloud-like enhancement” is a magnetic resonance imaging abnormality specific to neuromyelitis optica. Ann Neurol 2009;66:425-28 CrossRef Medline

11. Long $Y$, Chen $M$, Zhang B, et al. Brain gadolinium enhancement along the ventricular and leptomeningeal regions in patients with aquaporin-4 antibodies in cerebral spinal fluid. J Neuroimmunol 2014;269:62-67 CrossRef Medline

12. Pekcevik Y, Izbudak I. Perivascular enhancement in a patient with neuromyelitis optica spectrum disease during an optic neuritis attack. J Neuroimaging 2015;25:686-87 CrossRef Medline

13. Kim W, Park MS, Lee SH, et al. Characteristic brain magnetic resonance imaging abnormalities in central nervous system aquaporin-4 autoimmunity. Mult Scler 2010;16:1229-36 CrossRef Medline

14. Kim JE, Kim SM, Ahn SW, et al. Brain abnormalities in neuromyelitis optica. J Neurol Sci 2011;302:43-48 CrossRef Medline

15. Kim W, Kim SH, Lee SH, et al. Brain abnormalities as an initial manifestation of neuromyelitis optica spectrum disorder. Mult Scler 2011;17:1107-12 CrossRef Medline

16. McGraw CA, Swerdlow ML, Robbins MS. Acute reversible periependymal ventricular enhancement in neuromyelitis optica. Eur J Neurol 2012;19:57-58 CrossRef Medline

17. Cheng C, Jiang Y, Chen X, et al. Clinical, radiographic characteristics and immunomodulating changes in neuromyelitis optica with extensive brain lesions. BMC Neurol 2013;13:72 CrossRef Medline

18. Pekcevik Y, Orman G, Lee IH, et al. What do we know about brain contrast enhancement patterns in neuromyelitis optica? Clin Imaging 2016;40:573-80 CrossRef Medline
19. Weinshenker BG, Wingerchuk DM, Vukusic S, et al. Neuromyelitis optica IgG predicts relapse after longitudinally extensive transverse myelitis. Ann Neurol 2006;59:566-69 CrossRef Medline

20. Wingerchuk DM, Weinshenker BG. Neuromyelitis optica: clinical predictors of a relapsing course and survival. Neurology 2003;60: 848-53 CrossRef Medline

21. Keegan M, Pineda AA, McClelland RL, et al. Plasma exchange for severe attacks of CNS demyelination: predictors of response. $\mathrm{Neu}$ rology 2002;58:143-46 CrossRef Medline

22. Wingerchuk DM. Neuromyelitis optica: effect of gender. J Neurol Sci 2009;286:18-23 CrossRef Medline

23. Barnett Y, Sutton IJ, Ghadiri M, et al. Conventional and advanced imaging in neuromyelitis optica. AJNR Am J Neuroradiol 2014;35: 1458-66 CrossRef Medline

24. Argyriou AA, Makris N. Neuromyelitis optica: a distinct demyelinating disease of the central nervous system. Acta Neurol Scand 2008;118:209-17 CrossRef Medline

25. Nema N, Verma A, Choudhary U, et al. Ocular oscillations and transient oscillopsia in neuromyelitis optica. J Neurol Res 2014;4:146-50

26. Tahara M, Ito R, Tanaka $\mathrm{K}$, et al. Cortical and leptomeningeal involvement in three cases of neuromyelitis optica. Eur J Neurol 2012; 19:e47-e48 CrossRef Medline

27. Iorio R, Damato V, Mirabella M, et al. Distinctive clinical and neuroimaging characteristics of longitudinally extensive transverse myelitis associated with aquaporin-4 autoantibodies. J Neurol 2013; 260:2396-402 CrossRef Medline

28. Waters PJ, McKeon A, Leite MI, et al. Serologic diagnosis of NMO: a multicenter comparison of aquaporin-4-IgG assays. Neurology 2012;78:665-71; discussion 669 CrossRef Medline

29. Nicchia GP, Nico B, Camassa LM, et al. The role of aquaporin-4 in the blood-brain barrier development and integrity: studies in animal and cell culture models. Neuroscience 2004;129:935-45 CrossRef Medline

30. Vincent T, Saikali P, Cayrol R, et al. Functional consequences of neuromyelitis optica-IgG astrocyte interactions on blood-brain barrier permeability and granulocyte recruitment. J Immunol 2008; 181:5730-37 CrossRef Medline

31. Levy LM. Exceeding the limits of the normal blood-brain barrier: quo vadis gadolinium? AJNR Am J Neuroradiol 2007;28:1835-36 Medline

32. Bradl M, Lassmann H. Experimental models of neuromyelitis optica. Brain Pathol 2014;24:74-82 CrossRef Medline

33. Takahashi T, Fujihara K, Nakashima I, et al. Anti-aquaporin-4 antibody is involved in the pathogenesis of NMO: a study on antibody titre. Brain 2007;130:1235-43 CrossRef Medline

34. Sato DK, Callegaro D, de Haidar Jorge FM, et al. Cerebrospinal fluid aquaporin-4 antibody levels in neuromyelitis optica attacks. Ann Neurol 2014;76:305-09 CrossRef Medline

35. Jarius S, Franciotta D, Paul F, et al. Cerebrospinal fluid antibodies to aquaporin-4 in neuromyelitis optica and related disorders: frequency, origin, and diagnostic relevance. J Neuroinflammation 2010; 7:52 CrossRef Medline

36. Nishiyama S, Ito T, Misu T, et al. A case of NMO seropositive for aquaporin-4 antibody more than 10 years before onset. Neurology 2009;72:1960-61 CrossRef Medline

37. Bergamaschi R, Ghezzi A. Devic's neuromyelitis optica: clinical features and prognostic factors. Neurol Sci 2004;25(suppl 4):S364-67 CrossRef Medline

38. Lana-Peixoto MA. Devic's neuromyelitis optica: a critical review. Arq Neuropsiquiatr 2008;66:120-38 CrossRef Medline

39. Jarius S, Ruprecht K, Wildemann B, et al. Contrasting disease patterns in seropositive and seronegative neuromyelitis optica: a multicentre study of 175 patients. J Neuroinflammation 2012;9:14 CrossRef Medline

40. Jiao Y, Fryer JP, Lennon VA, et al. Updated estimate of AQP4-IgG serostatus and disability outcome in neuromyelitis optica. Neurology 2013;81:1197-204 CrossRef Medline

41. Bonnan M, Cabre P. Plasma exchange in severe attacks of neuromyelitis optica. Mult Scler Int 2012;2012:787630 CrossRef Medline 